\title{
DETECTION OF JAK2 MUTATION IN CASES OF MYELODYSPLASTIC SYNDROME
}

\author{
Mervat M. M. Azab, Ola A. Hussein and Mervat S.H. Amin \\ Clinical pathology Department, Faculty of Medicine, \\ Zagazig University, Egypt
}

\begin{abstract}
Background: JAK2V617F is a tyrosine kinase gain-of-function mutation in exon 14 that results from a guanineto-thymine transversion at nucleotide 1849 with substitution of valine to phenylalanine at codon 617 . The in vitro expression of JAK2V617F results in the constitutive activation of the JAK-STAT pathway and resultant cytokine-independent growth. Aim of the work: Detection of JAK2 mutation in cases of MDS and MDS/MPN to provide practical guidelines, which can allow for a reproducible classification of these types when encountered in clinical practice, and that will benefit of JAK2 inhibitor treatment. Patients and methods: This study comprised 40 newly diagnosed patients divided into two groups: Group I : 20 patients with MDS, Group II: 20 patients with MDS/MPN ( 2 cases aCML, 10 cases CMML, 8 cases JMML).All members of this study were subjected to the following :full history taking, complete clinical examination, routine laboratory investigations including:CBC, stained smear for morphological examination, B.M. aspirate \& examination, cytogenetic analysis and detection of JAK2 V617F mutation by Allele Specific PCR technique. Results: In typical forms of MDS JAK2 V617F mutation was very rare (5\%) However, a higher percentage of this mutation (55\%) was found in patients with MDS/MPN. Positive JAK2 patients with high TLC, LDH may be the main pathology but if negative JAK2 with high TLC, LDH and platelets, we should searched for other mutation that may be in other area of JAK family. Conclusion: our study stated that the presence of JAKV617F mutation may assist in diagnosis of MDS/MPN group than MDS group (odds ratio [OR], 23.2; 95\% confidence interval[CI], (2.5208.6); $\mathrm{P}=0.001$ ).
\end{abstract}

Key Words: JAK2 V617F mutation, MDS, MDS/MPN

\section{INTRODUCTION}

T he JAK family proteins (JAKs) are cytoplasmic tyrosine kinases that participate in cytokine receptor superfamily signaling, which transduce cytokine-mediated signals via the JAK-Signal Transducers and Activators of Transcription (STAT) pathway ${ }^{(\mathbf{1})}$.

The identification of the JAK2V617F mutation is an exciting new discovery in the field of chronic myeloproliferative disorders (MPDs) . This acquired mutation is characterized by a $G$ to $T$ transversion at nucleotide 1849 in exon 14 of the JAK2 gene, leading to a substitution of valine to phenylalanine at amino acid position 617 (V617F) of the JAK2 protein $^{(2)}$.

The presence of the mutation confers a proliferative and survival advantage by rendering the cells more sensitive to incoming stimulatory signals, causing clonal expansion of hematopoietic progenitors in myeloproliferative disorders. Neoplastic cells can be heterozygous for the mutation or hemizygous if they are associated with loss of heterozygosity (LOH) of 9p, where JAK2 is situated ${ }^{(3)}$.

Myelodysplastic syndromes (MDS) are a set of oligoclonal disorders of hematopoietic stem cells characterized by ineffective hematopoiesis that manifest clinically as anemia, neutropenia, and/or thrombocytopenia of variable severity. The result often is transfusion-dependent anemia, and increased risk of infection or hemorrhage, and a potential to progress to acute myelogenous leukemia (AML) ${ }^{(4)}$

The FAB classification served as the first framework to systematically identify and describe the different clinical entities of $\mathrm{MDS}^{(5)}$.World Health Organization (WHO) proposed a newer classification system attempting to address some of the limitations of the FAB system. The main goal of this schema was to formally incorporate biologic data, specifically cytogenetic and molecular findings, clinical outcomes, and number of dysplastic lineages involved, in order to better separate subgroups and delineate syndromes within the previous FAB classification ${ }^{(6)}$.After introduction of the WHO classification, WHO classification-based prognostic scoring system (WPSS) could classify patients into five risk groups showing different survivals and probabilities of leukemic evolution ${ }^{(7)}$.

In 2001 and 2008, the Word Health Organisation (WHO) revised the criteria for the 
classification of haematopoietic and lymphoid neoplasms in order to define better specific disease entities and to include significant prognostic information ${ }^{(8)}$.This classification recognises a new separated category of myelodysplastic/myeloproliferative disease (MDS/MPD) which consists of myeloid disorders with both dysplastic and proliferative features at the time of initial presentation and includes chronic myelomonocyic leukemia (CMML), atypical chronic myeloid leukemia (aCML), juvenile myelomonocyticleukaemia (JMML) and myelodysplastic/myeloproliferativediseaseunclassifi able(MDS/MPD-U) ${ }^{(9)}$.

Acquired JAK2V617F somatic mutation is a hallmark of Philadelphia negative myeloproliferative neoplasm (MPN) ${ }^{(\mathbf{1 0})} \cdot$ In myelodysplastic syndromes (MDS), JAK2V617F mutation is seen in less than $5 \%$ of the cases ${ }^{(11)}$. The JAK2V617F mutation was also found in patients with non classic MPN, although in much lower frequency: less than $20 \%$ of patients with aCML, less than $5 \%$ of patients with chronic myelomonocytic leukemia (CMML), less than $20 \%$ of patients with juvenile myelomonocytic leukemia (JMML) about 5\% of patients with MDS, and rarelyin patients with systemicmastocytosis ${ }^{(\mathbf{1 2})}$.

Aim of this work was to detect JAK2 mutation to provide practical guidelines, which can allow for a reproducible classification of these types of MDS and MDS/MPN when encountered in clinical practice, and that will benefit of JAK2 inhibitor treatment.

\section{PATIENTS AND METHODS}

This study was carried out at Clinical Pathology and Medical Oncology \& Hematology Departments, Faculty of Medicine, Zagazig University Hospitals. Forty newly diagnosed patients divided into two groups: Group I : 20 patients with MDS(4cases RA, 2 casesRT,10cases RCMD, 3cases RAEB, one case MDS-u) they were 8 male and 12 female their age ranged from 24-70 years with a mean age $54.8 \pm 11.14$ years. Group II: 20 patients with MDS/MPN ( 2 cases aCML, 10 cases CMML, 8 cases JMML) they were 12 male and 8 female their age ranged from 0.7-76 years with a mean age $38.3 \pm 31.24$ years.

Method: all members of this study were subjected to the following:full history taking , complete clinical examination, Routine laboratory investigations: $\mathrm{CBC}$ by " Advia 120" , stained smear for morphological examination, B.M. aspirate\& examination, cytogenetic analysis by $\mathrm{G}$ banding technique and LDH by "Dimension RxL Max". Specialinvestigations: detection of JAK2 V617F mutation by Allele Specific PCR technique. Genomic DNA was extracted from bone marrow aspirates or peripheral blood samples using Gspin $^{\text {TM }}$ Total DNA Extraction Kit manufactured by iNtRON biotechnology. The JAK2V617F mutation was detected according to the protocol of Baxter et al. ${ }^{(2)}$ with some modifications. Particularly, the primers used for multiplex PCR

* the primers used for multiplex PCR were $\mathrm{J} 1$ (wild-type sequence reverse primer)

$\rightarrow 5^{\prime}-$

CTGAATAGTCCTACAGTGTTTTCAGTTTCA3'

$\mathrm{J} 2$ (J1 a mutation-specific forward primer )

$\rightarrow 5^{\prime}-$

AGCATTTGGTTTTAAATTATGGAGTATATT3'

and $\mathrm{J} 3$ (forward-internal control)

$\rightarrow 5^{\prime}-$

ATCTATAGTCATGCTGAAAGTAGGAGAAAG $-3^{\prime}$

The thermal cycling procedure was performed with "Gene Amp PCR System 2400 "supplied by ( Perkin Elmer, USA) and consist of initial denaturation at $94{ }^{\circ} \mathrm{C}$ for 2 min.followed by 35 cycles $\left(94^{\circ} \mathrm{C}\right.$ for $45 \mathrm{~s}, 58^{\circ} \mathrm{C}$ for $45 \mathrm{~s}, 72^{\circ} \mathrm{C}$ for 60 $\mathrm{s}$ ), and $5 \mathrm{~min}$ at $72{ }^{\circ} \mathrm{C}$.The PCR products were analyzed on $2 \%$ TAE agarose gels. The primers $\mathrm{J} 1$ and J3 amplify a 364 bp product (both mutant and wild-type alleles and serves as an internal control), while the primers $\mathrm{J} 1$ and J2 amplify a $203 \mathrm{bp}$ product (when the patient carries the JAK2V617F mutation) ${ }^{(2)}$.

The data were tabulated and statistically analyzed using Microsoft Office Excel 2010 and Statistical package for Social Sciences version 20 (SPSS : An IBM Company).

\section{RESULTS}

The results of the present study are statistically analyzed and summarized in the following tables (1)-(7) and figure (1). 
Table (1): Clinical presentation of studied groups

\begin{tabular}{ccc} 
Clinical presentation & $\begin{array}{c}\text { Group I } \\
\text { No=20 } \\
\text { Number (\%) }\end{array}$ & $\begin{array}{c}\text { Group II } \\
\text { No=20 } \\
\text { Number (\%) }\end{array}$ \\
\hline Anaemia & $4(20 \%)$ & $3(15 \%)$ \\
\hline Anaemia+Bleeding & $4(20 \%)$ & $1(5 \%)$ \\
\hline Anaemia+Infection & $5(25 \%)$ & $1(5 \%)$ \\
\hline Infection+Bleeding & $1(5 \%)$ & $1(5 \%)$ \\
\hline Splenomegaly+anaemia & $6(30 \%)$ & $2(10 \%)$ \\
\hline Hepatomegaly+ anaemia & $0(0 \%)$ & $12(60 \%)$ \\
\hline
\end{tabular}

This table showed thatanaemic manifestation ( fatigue, pallor or fainting ) was (20\%) in group I and $(15 \%)$ in group II. Splenomegaly and anaemiawere (30\%) in group I and $(60 \%)$ in group II. Anaemia and repeated infection were $(25 \%)$ in group I and (5\%) in group II. Anemia and bleeding were (20\%) in group I and $(5 \%)$ in group II . Infection and bleeding were $(5 \%)$ in group I and (5\%) in group II finally hepatomegaly and anaemia were $(10 \%)$ in group II only.

Table (2): Laboratory data of studied groups.

\begin{tabular}{|c|c|c|c|c|}
\hline Parameter & $\begin{array}{l}\text { Group I } \\
(\mathrm{No}=\mathbf{2 0})\end{array}$ & $\begin{array}{l}\text { Group II } \\
(\mathbf{N o}=\mathbf{2 0})\end{array}$ & $\begin{array}{c}\text { Test of } \\
\text { significance } \\
\text { ( T ) }\end{array}$ & $P$ value \\
\hline \multicolumn{5}{|l|}{$\operatorname{TLC}\left(\mathrm{x}^{2} 0^{3} / \mu \mathrm{L}\right)$} \\
\hline Mean \pm SD & $11.23 \pm 5.1$ & $106.44 \pm 50.3$ & \multirow[b]{2}{*}{8.42} & \multirow[b]{2}{*}{$\begin{array}{c}0.001^{*} \\
\mathrm{HS}\end{array}$} \\
\hline Range & $1.5-41.6$ & $20.4-433$ & & \\
\hline \multicolumn{5}{|c|}{ Absolute monocytic count $\left(\mathrm{x10}^{3} / \mu \mathrm{L}\right)$} \\
\hline Mean \pm SD & $0.165 \pm 0.03$ & $25.844 \pm 9.1$ & \multirow{2}{*}{12.68} & $0.001^{*}$ \\
\hline Range & $0.1-0.3$ & $1.6-99.5$ & & H S \\
\hline \multicolumn{5}{|l|}{ Hemoglobin (g/dL) } \\
\hline Mean \pm SD & $8.82 \pm 1.98$ & $8.21 \pm 1.04$ & \multirow[b]{2}{*}{1.225} & \multirow{2}{*}{0.228} \\
\hline Range & $5.1-8.1$ & $7.2-10.5$ & & \\
\hline \multicolumn{5}{|l|}{ Platelet count $\left(\mathrm{x}^{3} 0^{3} / \mu \mathrm{L}\right)$} \\
\hline Mean \pm SD & $112.8 \pm 56.4$ & $84.8 \pm 42.4$ & \multirow[b]{2}{*}{1.351} & \multirow{2}{*}{0.083} \\
\hline Range & $9-685$ & $12-375$ & & \\
\hline \multicolumn{5}{|l|}{ BM blast (\%) } \\
\hline Mean \pm SD & $5.4 \pm 2.1$ & $4.15 \pm 2$ & \multirow[b]{2}{*}{1.732} & \multirow{2}{*}{0.061} \\
\hline Range & $1-18$ & $1-12$ & & \\
\hline \multicolumn{5}{|l|}{ LDH (IU/L) } \\
\hline Mean \pm SD & $511.4 \pm 220.8$ & $1652.5 \pm 826.1$ & \multirow{2}{*}{5.97} & \multirow{2}{*}{$\begin{array}{c}0.001^{*} \\
\mathrm{HS} \\
\end{array}$} \\
\hline Range & $233-945$ & $452-5635$ & & \\
\hline
\end{tabular}

This table showed that there was a highly significant difference between both groups as regards TLC, Absolute monocyte count and $\mathrm{LDH}(\mathrm{P}<0.01)$. 
Table (3): karyotyping of thestudied groups.

\begin{tabular}{|c|c|c|c|c|}
\hline \multirow{2}{*}{ Karyotype } & \multicolumn{2}{|c|}{$\begin{array}{c}\text { group I } \\
\text { No. }=\mathbf{2 0} \\
\end{array}$} & \multicolumn{2}{|c|}{$\begin{array}{l}\text { group II } \\
\text { No. }=\mathbf{2 0} \\
\end{array}$} \\
\hline & Frequency & $\%$ & Frequency & $\%$ \\
\hline Normal & 8 & $40 \%$ & 9 & $45 \%$ \\
\hline del 5q & 3 & $15 \%$ & - & - \\
\hline del 20q & 2 & $10 \%$ & - & - \\
\hline+8 & 2 & $10 \%$ & 1 & $5 \%$ \\
\hline$-7 /$ del7q & 1 & $5 \%$ & - & - \\
\hline $\mathbf{i}(17)(q 10)$ & - & - & 2 & $10 \%$ \\
\hline+21 & - & - & 3 & $15 \%$ \\
\hline$-7 / \operatorname{del} 7 q / \operatorname{del}(11 q)$ & 1 & $5 \%$ & - & - \\
\hline$+21 / \operatorname{del}(20 \mathrm{q}) /-7$ & - & - & 1 & $5 \%$ \\
\hline Unavailabe & 3 & $15 \%$ & 4 & $20 \%$ \\
\hline
\end{tabular}

This table showed that cytogenetics finding of group I, 40\% had normal karyotype, $15 \%$ had del(5q), $10 \%$ had del(20q), 10\% had trisomy $8,5 \%$ had 7/del(17q),5\% had complex abnormalities $-7 /$ del7q/ del(11q) ,15\% were unavilable. While cytogenetics finding of group II, $45 \%$ had normal cytogenetics, $15 \%$ had Trisomy $21,10 \%$ had i(17)(q10),5\% had trisomy $8,5 \%$ had complex abnormalities in the form of $+21 /$ del(20q)/-7, $20 \%$ were unavilable.

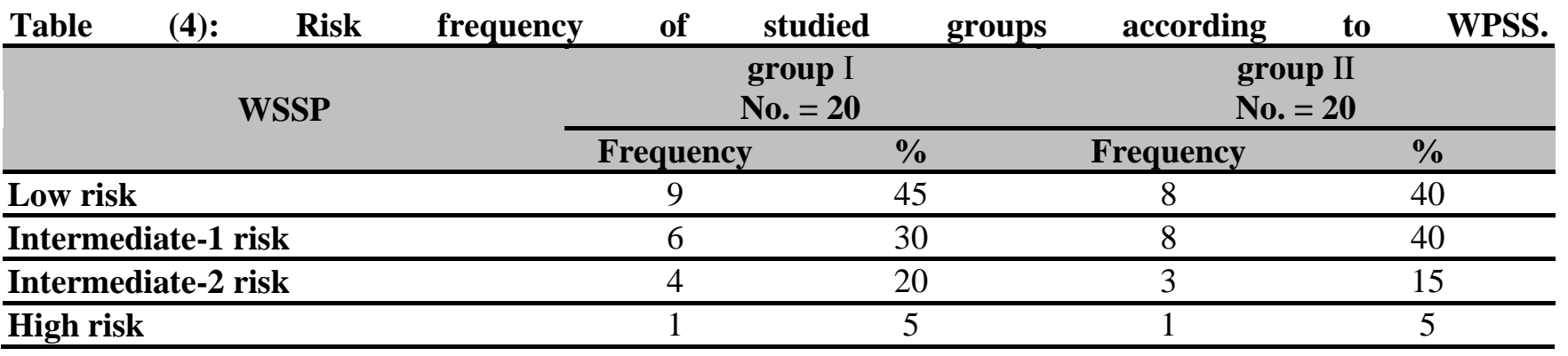

Among grouplaccording to WPSS system, 9 patients (45\%) were classified as low risk, 6 patients (30\%) were classified as Intermediate-1 risk, 4 patients (20\%) were classified as Intermediate-2 risk, one patient (5\%) was classified as High risk, .While among group Ilaccording to WPSS system, 8 patients $(40 \%)$ were classified as low risk, 8 patients $(40 \%)$ were classified as Intermediate-1 risk, 3 patients (15\%) were classified as Intermediate- 2 risk, one patient $(5 \%)$ were classified as High risk.

Table (5): PCR result of JAKV617F mutation in studiedgroups.

\begin{tabular}{ccc}
\hline \multirow{2}{*}{ Group } & \multicolumn{3}{c}{ JAK2 V617F } \\
\cline { 2 - 3 } & Positive cases & Percent \\
\hline Group I & 1 & $5 \%$ \\
\hline Group I & 11 & $55 \%$ \\
\hline
\end{tabular}

This table revealed that JAK2V617F mutation was detected in one case(5\%) of group I and eleven cases (55\%)of group II. 
Table (6): Comparsion between JAK positive and JAK negative cases as regard age, TLC, HB, Platelets , absolute monocyte count (AMC), B.M. blast, LDH and WPSS.

\begin{tabular}{ccccc}
\hline & $\begin{array}{c}\text { JAK2 positive } \\
\mathbf{n o}=\mathbf{1 2}\end{array}$ & $\begin{array}{c}\text { JAK2 negative } \\
\mathbf{n o = 2 8}\end{array}$ & $\begin{array}{c}\text { Test of } \\
\text { significance } \\
\text { ( } \mathbf{~}\end{array}$ & P value \\
\hline Age (years) & $40.78 \pm 20.27$ & $49.03 \pm .24 .5$ & 1.04 & .312 \\
\hline TLC $(\mathbf{x 1 0 3 / \mu L )}$ & $78.97 \pm 39.2$ & $50.20 \pm 21.1$ & 1.4 & .145 \\
\hline HB $(\mathbf{g} / \mathbf{d L})$ & $8.04 \pm 0.96$ & $8.7 \pm .1 .77$ & -1.242 & 0.222 \\
\hline Platelets $(\mathbf{x 1 0} / \boldsymbol{\mu L})$ & $27.75 \pm 13.9$ & $129.28 \pm 64.6$ & 3.9 & $0.008^{*}$ \\
\hline AMC $(\mathbf{x 1 0} / \boldsymbol{\mu L})$ & $23.01 \pm 11.5$ & $8.71 \pm 4.3$ & 1.18 & 0.063 \\
\hline B.M. blast $(\%)$ & $6.16 \pm .3 .26$ & $2.03 \pm .1 .68$ & 4.34 & $0.0001^{*}$ \\
\hline LDH $(\mathbf{I U} / \mathbf{H})$ & $2104.2 \pm 852.1$ & $643.8 \pm 121.9$ & 5.91 & $0.001^{*}$ \\
\hline WPSS & $1.20 \pm .0 .75$ & $1.107 \pm .0 .59$ & 0.453 & 0.653 \\
\hline
\end{tabular}

This table revealed that there was a significant difference between JAK2 positive and JAK2 negative cases as regards platelet count $(\mathrm{P}<0.05)$. There was a highly significant difference between JAK2 positive and JAK2 negative cases as regards bone marrow blast and LDH $(\mathrm{P}<0.01)$.

Table (7): Comparsion between JAK positive and JAK negative patients as regard presence of splenomegaly.

\begin{tabular}{ccccccccc}
\hline \multirow{2}{*}{ Splenomegaly } & \multicolumn{2}{c}{ JAK2 positive } & \multicolumn{2}{c}{ JAK2 negative } & \multicolumn{2}{c}{ Total } & \multicolumn{2}{c}{ P value } \\
\cline { 2 - 7 } & Frequency & $\%$ & Frequency & $\%$ & Frequency & $\%$ & \\
\cline { 1 - 7 } Splenomegaly & 11 & 91.7 & 7 & 25 & 18 & 45 & \multirow{2}{*}{$0.0001 *$} \\
\hline No Splenomegaly & 1 & 8.3 & 21 & 75 & 22 & 55 & HS \\
\hline Total & 12 & 100 & 28 & 100 & 40 & 100 & \\
\hline
\end{tabular}

There was a highly significant difference between JAK2 positive and JAK2 negative patients as regards splenomegaly $(\mathrm{P}<0.01)$.

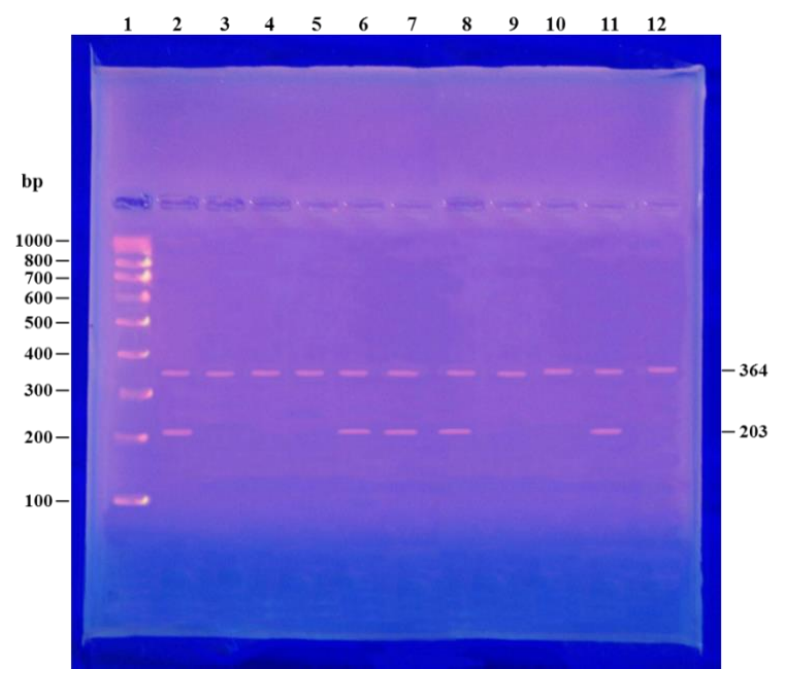

Figure(1): Gelelectrophoresis of PCR products for JAK2V617F mutation established by allele-specific PCR . Lane 1: 100-1000 bp ladder molecular weight marker. Lane 2, 6,7,8,11 DNA of patients samples positive for JAK2V617F mutation show amplification products at 203, $364 \mathrm{bp}$ (mutant allele), while other lanes are negative for JAK2 V617F mutation showed only single amplification product at $364 \mathrm{bp}$ (unmutant wild type allele). 


\section{DISCUSSION}

Myelodysplastic syndromes (MDS) constitute a heterogeneous group of clonal hematopoietic disorders characterized by bone marrow (BM) failure, dysplasia and an increased risk of developing acute myeloid leukemia $(\mathrm{AML})^{(\mathbf{1 3})}$.

The myelodysplastic/myeloproliferative neoplasms (MDS/MPN) include clonal myeloid neoplasms that overlap the MDS and MPN categories and at the time of initial diagnosis exhibit some clinical, laboratory, or morphologic features supporting the diagnosis of myelodysplastic syndrome (MDS) and at the same time show proliferative features in keeping with the diagnosis of a myeloproliferative neoplasm (MPN) ${ }^{(14)}$.

Since the publication of the WHO classification in 2008, dynamic progress in array technologies and next-generation amplicon deep sequencing has provided new insights into the molecular pathogenesis of MDS/MPN ${ }^{(\mathbf{1 4})}$.

The in vitro expression of JAK2V617F results in the constitutive activation of The JAK-STAT pathway and resultant cytokine-independent growth. Acquired JAK2V617F somatic mutation is a hallmark of Philadelphia negative myeloproliferative neoplasm (MPN) ${ }^{(\mathbf{1 0})}$.

The objective was to detect JAK2 mutation in cases of MDS and MDS/MPN to provide practical guidelines, which can allow for a reproducible classification of these types of MDS and MDS/MPN when encountered in clinical practice, and that will benefit of JAK2 inhibitor treatment.

This Study was carried out on 40 newly diagnosedpatients classified into two groups: group I (20 MDS patients), group II (20 MDS/MPN patients).In this study There was statistically significant difference between the two groups as regard age $(\mathrm{P}<0.05)$ may be due to inclusion of young patients diagnosed as (JMML). There was no statistically significant difference between the two groups as regards gender $(\mathrm{P}>0.05)$ with male/female ratio $2: 3$. Our results contradict the results reported by Goldberg et al. ${ }^{(15)}$ they found that the mean age was 64.4 years and its range from 17.0 - 96.4 years with male/female ratio 1.51:1 .

In our study there was a highly significant increased TLC in group $\Pi$ when compared to group I . This was expected since leucopenia discriminates MDS from MDS/MPN in which there was some features of myeloproliferation. Monocytosis was highly significant in group II that include CMML and JMML. LDH was highly significant in group IIwhich is a character of myeloproliferation. LDH level can be a point of differentiation between MDS and MDS/MPN cases.

Our finding in this study showed that in MDS/MPN group, JAK2V617F mutation present in $10 \%$ of patients with a typical chronic myeloid leukemia (a CML), 30\% of patients with chronic myelomonocytic leukemia a(CMML), $15 \%$ of patients with juvenile myelomonocytic leukemia (JMML), while 5\% in MDS patients(data not showen). It was obvious that JAK2 V617F mutation appeared to be infrequent finding in MDS group and this was in agreement with the finding of Steensma et al. ${ }^{(16)}$, Jelinek et al. ${ }^{(17)}$ andBacher et al. ${ }^{(\mathbf{1 8})}$ they reported positivity rates JAK2V617F mutation to be 5\%, $1 \%, 3 \%$ respectively.Our results in groupIIshowed higher occurrence of JAK2V617F mutation in patients with MDS/MPN than MDS and this was in agreement with the finding of Jekarl et al. ${ }^{\left({ }^{(19)}\right.}$ who stated that $6(13.9 \%)$ of 43 patients, harbored the JAK2V617F mutation,the incidence of the JAK2V617F mutation in each of patient group was as follows: $8.3 \%$ (1/12), MDS; $22.2 \%$ (2/9), MDS/MPN-U; $14.3 \%$ (1/7), RARS-T; and $13.3 \%$, (2/15) AML.

In the present study there was a significant difference between JAK2 positive and negative cases as regards platelet count $(\mathrm{P}<0.05)$, as it was significantly low in JAK2 positive cases. This was explained by high incidence of splenomegaly as eleven patients of JAK2 positive had enlarged spleen and subsequently hyperslenism, which may be a cause of reduction in platelet count, in addition to our patients included in this study did not include RARS-T which characterized by high platelet count.

Also there was a highly significant difference between JAK2 positive and negative cases as regards bone marrow blast, LDH and splenomegaly $(\mathrm{P}<0.01)$. Our results showed that TLC was higher in JAK2 positive cases when compared with JAK2 negative cases, this mean that this mutation may be the main pathology and treatment with JAK2 inhibitors could be valuable. Patients with high TLC, LDH and positive JAK2 may be the main pathology but if negative JAK2 with high TLC, LDH and platelet in absence of splenomegaly, we should searched for other mutation that may be in other area of JAK family. 
Our finding contradict the results reported by Renzis et al. ${ }^{(20)}$ who stated that in JAK2 positive patients, the white blood cells (WBC) count, absolute neutrophil count (ANC), platelet count and mean corpuscular volume (MCV) were significantly higher, while the marrow blast percentage wassignificantly lower than in JAK2 negative cases.

While there was no significant differences between JAK2 positive and negative cases as regard age, gender, hemoglobin level, WHO classification, cytogenetic findings and WPSS.

Our results are in agreement with study of Renzis et al. ${ }^{(\mathbf{2 0})}$ who stated that there was no significant differences between JAK2 positive and negative cases as regard age, gender, hemoglobin level, WHO 2008 classification, cytogenetic findings and WPSS.

Our study stated that the presence of JAKV617F mutation may assist in diagnosis of MDS/MPN group than MDS group (odds ratio $[\mathrm{OR}], 23.2 ; 95 \%$ confidence interval[CI], (2.5208.6); $\mathrm{P}=0.001$ ).

Conclusion: The detection of the JAK2 V617F mutation, in difficult-to-classify cases, will help to clarify the borderline between MDS, on one hand, and MDS/MPN on the other hand. The better understanding of these entities, potentially may lead in the near future to identify new therapeutic options for these patients .

Recommendation: Multicenter studies are needed to develop a cytogenetic-molecular-based risk stratification system for optimal targeted therapy and improved outcome of MDS and MDS/MPN, as already used in AML management. Many more JAK inhibitors need to be evaluated in order to identify the best in class in terms of efficacy, toxicity and suitability for future combination treatment programs.

\section{REFERENCES}

1- Ward AC, Touw I and Yoshimura A (2000):The JakStat pathway in normal and perturbed hematopoiesis. Blood;95:19-29.

2- Baxter EJ, Scott LM, Campbell P, et al. (2005): Acquired mutation of the tyrosine kinase JAK2 in human myeloproliferative disorders. Lancet ;365:1054-1061.

3- Kralovics R, Passamonti F, Buser AS, et al. (2005): A gain-of-function mutation of JAK2 in myeloproliferative disorders. $\mathrm{N}$ Engl J Med;352:1779-1790.

4- Daniel VT,Catenacci, Gary J and Schiller (2005):Myelodysplasic syndromes: A comprehensive reviewBlood Reviews, 301-319.
5- Steensma DP and Bennett JM (2006): The myelodysplastic syndromes: diagnosis and treatment. Mayo Clin. Proc; 81: 104-130. 6- Harris NL, Jaffe ES, Diebold J, et al. (1999): World Health Organization classification of neoplastic diseases of the hematopoietic and lymphoid tissues: report of the Clinical Advisory Committee meeting-Airlie House, Virginia, J. Clin. Oncol., 17: 3835-3849.

7- Malcovati L, Germing U, Kuendgen A, et al.(2007) : Time-dependent prognostic scoring system for predicting survival and leukemic evolution in myelodysplastic syndromes. J ClinOncol 25(23):3503-10.

8- Jaffe E, Harris N, Stein H and Vardiman J.(2001): The World Health Organization classification of tumours: pathology and genetics of tumours of haematopoietic and lymphoid tissues .Lyon:IARC Press. 115:45244532.

9-Vardiman JW, Harris NL and Brunning RD(2002): The World Health Organization (WHO) classification of the myeloid neoplasms. Blood 100(7):2292-302 . 10- James C, Ugo V, Le Couedic JP, et al. (2005):A unique clonal JAK2 mutation leading to constitutive signaling causes polycythaemiavera. Nature;434:1144-1148.

11- Schmitt-Graeff AH, Teo S-S, Olschewski M, et al.(2008): JAK2V617 mutation status identifies subtypes of refractory anemia with ringed sideroblasts associated with marked thrombocytosis. Haematologica ;93:34-40.

12- Ohyashiki K, Ohyashiki JH. Kremer M et al. (2006): The JAK2 V617F mutation occurs frequently in myelodysplastic/myeloproliferative diseases, but is absent in true myelodysplasticsyndromeswith fibrosis. Leukemia ;20:1297-1298.

13- Platzbecker U, Santini V, Ghulam J, et al. (2012): Update on developments in the diagnosis and prognostic evaluation of patientswith myelodysplastic syndromes (MDS): Consensus statements and report froman expert workshop Leukemia Research 36 264-270.

14- Elizabeth $\mathrm{H}$ and James W. V (2011):Myelodysplastic/myeloproliferative neoplasmsSeminars in Diagnostic Pathology, Volume 28, Issue 4, Pages283-297.

15-Goldberg SL, Chen E, Corral M, Guo A, Mody-Patel N, Pecora AL, Laouri M.(2010):Incidence and clinical complications of myelodysplastic syndromes among United States Medicare beneficiaries. J ClinOncol. Jun 10;28(17):2847-52. doi: 10.1200/JCO.2009.25.2395. Epub 2010 Apr 26.

16- Steensma, D.P., Dewald, G.W., Lasho, T.L., Powell, H.L., McClure, R.F., Levine, R.L., Gilliland, D.G.\&Tefferi, A. (2005):The JAK2 V617F activating tyrosine kinase mutation is an infrequent event in both "atypical" myeloproliferative disorders and myelodysplastic syndromes. Blood, 106, 1207-1209. 
17-JelinekJ,OkiY,Gharibyan V, et al. (2005):JAK2 mutation $1849 \mathrm{G} 4 \mathrm{~T}$ is rare in acute leukemias but can be found in CMML, Philadelphia-chromosome negative CML and megakaryocytic leukemia. Blood 2005; 106: 3370-3373.

18-Bacher U, Schnittger S, Kern W, Weiss T,Haferlach T. and Haferlach C. (2009):Distribution of cytogenetic abnormalities in myelodysplastic syndromes, Philadelphia negative myeloproliferative neoplasms, and the overlap MDS/MPN category Ann Hematol 88:1207-1213.

19-Jekarl DW, Han SB, Kim M, Lim J, Oh EJ, Kim Y, Kim HJ, Min WS, and Han K (2010):JAK2V617F mutation in myelodysplastic syndrome, myelodysplastic syndrome/myeloproliferative neoplasm, unclassifiable, refractory anemia with ring sideroblasts with thrombocytosis, and acute myeloid leukemia. Korean J Hematol. Mar;45(1):46-50.

20-Renzis B , De Mas V M, Wattel E, Beyne-Rauzy O, Knoops L, Cabrespine A, Azgui Z , Ades L and KiladjianJ(2013): Prognostic impact of JAK2V617F mutation in myelodysplatic syndromes: A matched case control study Leukemia Research Reports $86: 64-66$ 